\title{
Toward a mechanistic understanding of wettability alteration in reservoir rocks using silica nanoparticles
}

\author{
Saeed Jafari Daghlian Sofla ${ }^{1}$, Lesley Anne James ${ }^{1, *}$, Yahui Zhang ${ }^{1}$ \\ ${ }^{1}$ Memorial University of Newfoundland, St. John's, Canada
}

\begin{abstract}
Traditional concepts of simple liquid spreading may not apply to nanoparticle-fluids. Most investigations pertaining to the wettability alteration of solid surfaces due to the presence of nanoparticles in the fluid are oversimplified, i.e. nanoparticles dispersed in DI-water and smooth, homogeneous, and clean surfaces have been used. From a practical enhanced oil recovery (EOR) point of view, the nanoparticles must be dispersed in either seawater or high salinity formation water containing diverse types and concentrations of ions. These ions interact with the electrostatic properties of the nanoparticles. Likewise, the oil phase may contain many surface active components like asphaltene and naphthenic acids which can interact with nanoparticles at oil-water and oil-rock interface. In reality, the rock sample is a heterogeneous, non-smooth, mixed-wet substrate with a diverse mineralogical composition. The electrical charge of minerals can vary when contacted with an ionic fluid. This can alter the electrostatic repulsion between substrate and nanoparticles and consequently the substrate can either attract or repel charged particles, including nanoparticles. Hence, the role of nanoparticles must be evaluated considering multicomponent complex fluids and real formation rock. Despite numerous reports regarding the wettability alteration of reservoir rock from oil-wet to water-wet by nanoparticles, some inherent limitations in the wettability alteration experiments prevent conclusions about the performance of nanoparticles in practical complex conditions. For instance, the wettability alteration by nanoparticles is often determined by contact angle measurements. In this method, the substrates are either aged with (immersed in) nanoparticle-fluids before conducting the experiments or contacted with nanoparticle-fluids before attachment of the oil droplet on the rock surface. Hence, in both cases, before initiating the contact angle measurements, the nanoparticles would already exist at the oil-rock interface possibly giving inaccurate measurements. The objective of this work is to investigate the mechanism of wettability alteration by silica nanoparticles pre-existing on the rock interface (conventional contact angle measurements) and using a new displacement contact angle method to better mimic the scenario of injecting a nanoparticle fluid into the reservoir already containing formation brine. The impact of pre-existing nanoparticles at the oil-rock interface (in the conventional contact angle measurements) on the contact angle measurements are examined for simple (n-decane, $\mathrm{NaCl}$ brine, and pure substrates) and complex (crude oil, seawater, and reservoir rock) systems on various wetting conditions of substrates (water-wet and oil-wet). The nanoparticles are dispersed in seawater using our $\mathrm{H}+$ protected method [1]. Then, the effect of surface and nanoparticle charge on the contact angle is evaluated by adjusting the aqueous phase salinity. We also differentiate between the disjoining pressure mechanism and diffusion of silica nanoparticles through the oil phase by testing the attachment of nanoparticles on the rock surface.
\end{abstract}

\section{Introduction}

Utilizing a modified aqueous phase to displace immobile oil is one method to improve pore scale recovery during some chemical EOR methods such as smart water, low salinity water injection [2], surfactant flooding [3], and potentially for nanoparticle enhanced water flooding [4]. This has led to a growing interest in understanding mechanism of oil removal from a solid surface in the presence of an aqueous solution containing different ionic strengths [5], micellar solutions $[6,7]$ and/or nanoparticles [8-10]. Kao et al. [7] suggested the combination of "diffusion" and "roll-up" mechanisms to remove oil from a solid surface in the presence of a micellar solution. They mentioned that the receding of solid-oil-water contact angle is caused by the "roll-up" mechanism and the presence of the second contact line is due to "diffusion". Roll-up, as shown in Figure 1a, can be simply defined as the removal of an oil droplet from a rock surface due to the decrease in contact angle between the aqueous phase and the substrate. Miller and Raney [11] presented the "solubilization-emulsification"

* corresponding author: ljames@mun.ca 
mechanism for oil removal from oily soils. In this mechanism, as shown in Figure 1b, some portion of oil drop is detached from the surface by destabilizing the oilwater interface (reducing oil-water interfacial tension).

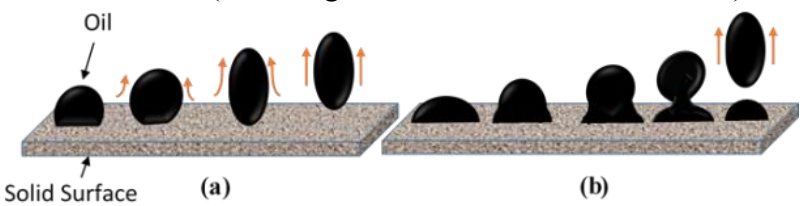

Fig. 1. Mechanism of oil removal from a surface by a)roll-up , and b) emulsification (after [11])

Wettability alteration due to nanoparticles (nanoparticlefluids) is complex. The conventional concepts of simple liquid spreading [12], due to the complex interactions between the nanoparticles and the solid surface at the three phase contact region, do not apply to nanoparticlefluids [9]. Kondiparty et al. [10] experimentally evaluated the dynamics of nanoparticle-fluids spreading by directly observing the self-layering of nanoparticles. They reached the conclusion that the three-phase contact line spontaneously decreases to reach an equilibrium condition. Then, nanoparticles form ordered structures in the confinement of the three-phase contact region. This ordering in the wedge-like area causes an extra pressure in the film compared to bulk solution and separating oil drop from the surface, as illustrated in Figure 2. This pressure is known as "structural disjoining pressure". Sefiane et al. [13] reported that the change in the contact angle of oil, water, and rock surface can be due to combination of "structural disjoining pressure" and "adsorption" of nanoparticles on the rock's surface.

Wedge-film

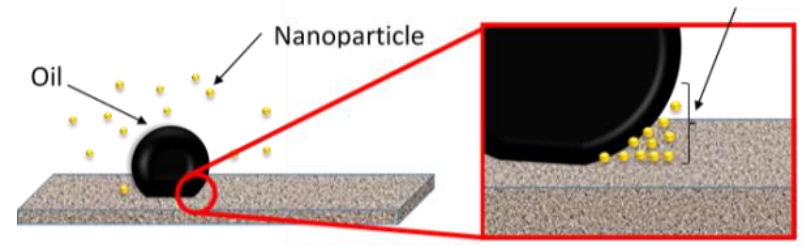

Fig. 2. Nanoparticle assembling in wedge film causes to structural disjoining pressure (After [14])

The wettability alteration of substrates using nanoparticle-fluids is sensitive to many factors including: nanoparticle size and concentration, initial contact angle [14], particle charge, surface wettability of nanoparticles [15], charge and roughness of the substrate surface, stabilizer concentration, type and concentrations of ions in the nanoparticle-fluids, bulk pressure and temperature, etc. Wasan et al. [14] tested canola oil drop spreading on a glass surface when surrounded by a silica nanoparticlesfluid. They pointed out that by increasing the concentration of nanoparticles, the structural disjoining pressure and spreading rate of the nanoparticles-fluid increases. They also noticed that the spreading rate of nanoparticles-fluid decreased with a decrease in the drop volume. Analyzing the effect of the contact angle on the shape of the meniscus profile illustrates that by altering the contact angle from $4^{\circ}$ to $2.3^{\circ}$ the more pronounced nominal contact line displacement is observed, and the drop detached from the surface at $2.3^{\circ}$. They did not report the accuracy and precision of their experiments. Wang and $\mathrm{Wu}[15]$ examined the effect of particle charge and surface wettability of the nanoparticles on oil drop detachment from a surface by using molecular dynamic simulation. Their simulation showed that full detachment of oil droplet from a solid surface by nanoparticles is possible when the charge of particles exceeds a threshold value. They concluded that high charged hydrophobic nanoparticles have the best performance in oil detachment. Lim et al. [16] demonstrated that an oil drop detaches faster when the temperature and hydrophilicity of the substrate increases. In all these studies, the system was oversimplified i.e. nanoparticles were dispersed in DI-water and the substrate was a smooth, clean and strongly hydrophilic surface. From an EOR point of view, wettability alteration of reservoir rock, and finally detachment of oil drop from a rock surface, is complex and oversimplified models may not fully mimic the practical EOR conditions. In the EOR processes, nanoparticles would be dispersed in either seawater or high salinity formation water containing diverse types and concentration of ions. The oil phase contains many polar and nonpolar components which can affect the performance of nanoparticles. The substrate is a heterogeneous, non-smooth, mixed-wet rock composed of various minerals. Furthermore, the existence of diverse minerals on the rock surface means that there are different electrostatic charges on the rock surface when contacted with an ionic solution.

Despite reports on wettability alteration of reservoir rock from oil-wet to water-wet by nanoparticles [17-20], some inherent limitations in the contact angle measurements may prevent researchers from concluding about the performance and mechanism of wettability alteration by nanoparticles under realistic conditions. For instance, the wettability alteration by nanoparticles is normally determined by apparent contact angle measurements. In this method, the substrates used are either used as-is or aged when the contact angle measurements are made. The aged substrates are submerged in the nanoparticle-fluid for 2-3 hours [21] or 2 days [22] before conducting the contact angle measurements. The unaged or as-is substrates are not aged but they are exposed to nanoparticle-fluid in the cell of the pendant drop apparatus before an oil droplet is attached to the surface (to conduct the contact angle measurement) [23-26]. In both cases as shown in Figure 3, in conventional contact angle measurements, the nanoparticles would already exist at the interfaces in both cases and possibly giving inaccurate measurements. However, under practical injection conditions, nanoparticles would not exist at the oil-rock interface before nanoparticle-fluids injection. Hence, the measured contact angles are not representative of practical injection conditions. 


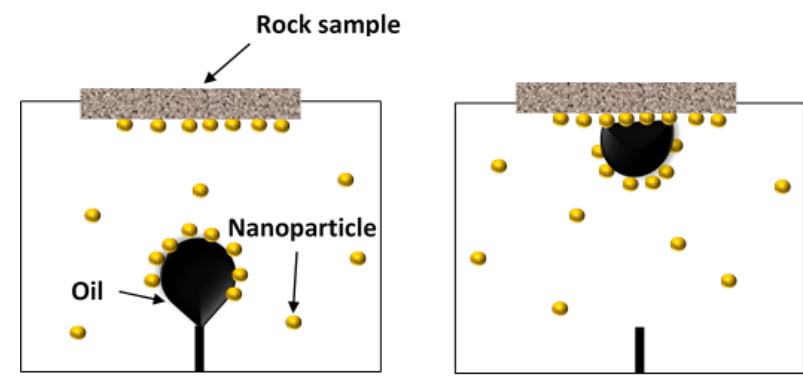

Fig. 3. Nanoparticles at oil-rock interface during conventional contact angle measurements

In this study, the mechanism of wettability alteration by silica nanoparticles is investigated. The experiments are designed in a way to avoid inherent problems associated with conventional contact angle measurements. The effect of adsorbed nanoparticles at the oil-rock interface on the ultimate contact angle values in the simple (toluene, nanoparticles dispersed in $1 \mathrm{wt} \% \mathrm{NaCl}$, and pure minerals) and complex system ( $35^{\circ} \mathrm{API}$ offshore NL crude oil, $\mathrm{H}+$ protected nanoparticles in seawater, and reservoir rocks) with various initial wetting conditions (water-wet, and oil-wet) are examined. Then, the effect of nanoparticles and substrate charge on the wettability alteration performance of silica nanoparticles is investigated. Finally, the migration of silica nanoparticles through a continuous oil phase and its attachment at the rock surface is tested. Based on the results, the mechanism of wettability alteration by silica nanoparticles is discussed.

\section{Materials}

Nanoparticle-fluids: Amorphous hydrophilic silicon dioxide nanoparticles $\left(\mathrm{SiO}_{2}\right)(25 \mathrm{wt} . \%$ in deionized [DI] water) with the average diameter of $19.0 \pm 0.8 \mathrm{~nm}$ and purity of greater than $99.9 \%$ were supplied by US Research Nanomaterial, Inc. Nanoparticle-fluids are prepared by dispersing $0.15 \mathrm{wt} \%$ of silica nanoparticles in either $\mathrm{NaCl}$ brine (simple system) or seawater using $\mathrm{H}+$ protected method [1] (complex system). Based on this method, $\mathrm{H}+$ ions prevent the multivalent ions in seawater to accumulate in the electrical double layer of silica nanoparticles by forming an $\mathrm{H}+$ layer at electrical double layer and thus stabilizing them in seawater.

Oil phase: Decane (simple system) and $35^{\circ} \mathrm{API}$ offshore NL crude oil (complex system) are used as the two oil phases.

Rock Samples: For the simple system, pure calcite, dolomite, and quartz are used as rock samples. For the complex system, unpolished chalk, carbonate rock and Berea sandstone are used. The size of the samples were approximately $1 \times 1 \mathrm{~cm}^{2}$. To obtain oil-wet samples, we aged the rocks in the crude oil at $60{ }^{\circ} \mathrm{C}$ for four weeks [27].

\section{Methods}

Contact angle measurements: As discussed before, the inherent limitations associated with conventional contact angle measurements can cause us to obtain misleading results. Hence, we modified the contact angle measurements in a way to have more consistency with practical EOR conditions. As shown in Figure 4, in real reservoir, first, the oil droplet is attached at rock surface while formation water is initially covering the surface. Then, the formation water is displaced by nanoparticlefluids. To mimic this condition in contact angle measurements, first the solid surface is contacted with a nanoparticle-free aqueous phase, and an oil drop (with the volume of $3-5 \mathrm{~mm}^{3}$ ) is injected from the needle at the bottom of the cell. The drop is attached onto the rock surface and its side image is taken at a frequency of one image per second. After equilibrium (approximately 20 min after forming the drop based on dynamic contact angle measurements), the apparent contact angle $(\theta)$ is measured. This contact angle indicates the initial state of oil droplet in the reservoir. Then, the nanoparticles-fluid is injected very slowly into the measurement cell to displace the aqueous phase (displacement method). The water is displaced slowly enough to not dislodge the oil droplet. To ensure that the aqueous phase is completely displaced by the nanoparticle-fluid, the turbidity of outlet fluid is frequently measured and compared with the turbidity of original nanoparticle-fluid. When the turbidity of outlet fluid and the original nanoparticle-fluid are equal, we assume that the aqueous phase has been completely displaced by the nanoparticle-fluid. All experiments are conducted at ambient conditions. To check the repeatability of measurements, three distinct measurements are performed, and the average is reported.
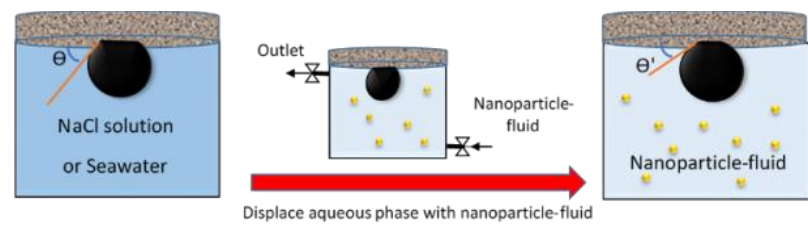

Fig. 4. Schematic diagram of modified contact angle measurements procedure: a) measure contact angle of oil-watersubstrate, b) displace aqueous phase with the nanoparticle-fluid, and c) measure the contact angle of oil-nanoparticle-fluidsubstrate

Zeta-potential measurements: The zeta-potential of substrates and nanoparticles in various aqueous solutions are measured by a Malvern Zetasizer Nano Series ZS instrument. To measure the zeta-potential of rock surfaces, the rock samples are milled and $1 \mathrm{wt} \%$ of milled rocks are dispersed in aqueous solutions and mixed with magnetic mixer for 30 minutes. Then the zeta-potential of samples are measured as described in [28].

Diffusivity experiments: A series of experiments are designed to detect the possible migration of silica nanoparticles through oil phase. As shown in Figure 5, the rock sample is located at the top of the cell in the contact with a layer of oil (the oil thickness is chosen arbitrarily 
as $1 \mathrm{~mm}$ ) at ambient conditions. First, the cell is filled with the aqueous phase (nanoparticle-free) and after three weeks, the images of the substrate contacted with an oil phase are taken by scanning electron microscope (SEM). Then, the aqueous phase is replaced by the nanoparticlefluid and after three weeks, another SEM image is taken from the same surface to detect possible nanoparticle attachments on the rock surface.

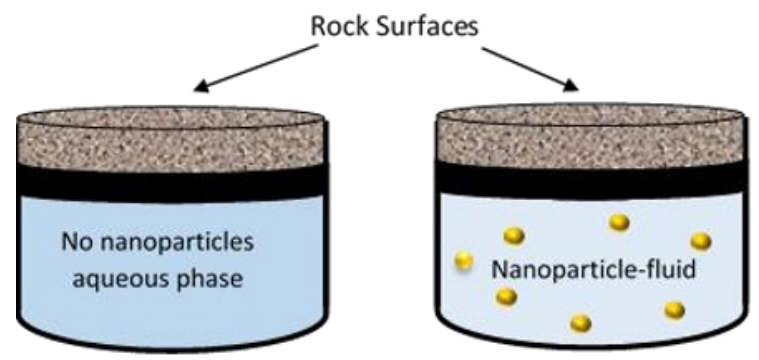

Fig. 5. Schematic diagram of diffusivity detection cells

\section{Results and discussion}

The impact of the experimental method on contact angle measurements for simple and complex systems is illustrated in Figure 6 and Figure 7, respectively. For each system, the apparent contact angle is measured for the three cases: nanoparticle-free fluid, nanoparticle fluid with the new displacement method, and nanoparticle fluid with the conventional methods. The initial conditions of the rocks are modified in a way to have an oil-wet and a water-wet surface for each rock type. For water-wet simple system, as shown in Figure 6a, the initial contact angle (without nanoparticles) for dolomite, quartz and calcite are measured within the aqueous phase (in degrees) as $70.8 \pm 1.5,77.6 \pm 1.8$ and $55.6 \pm 1.7$, respectively. By introducing silica nanoparticles in the system using the displacement method, the contact angles for three mentioned substrates decrease to $56.3 \pm 1.7,73.7$ \pm 1.8 , and $47.4 \pm 2.3$, respectively. On the other hand, by adding nanoparticles using the conventional contact angle measurement method, the contact angles reduce to $47.5 \pm$ $1.5,64.5 \pm 1.6$, and $37.9 \pm 2.0$, respectively. The difference in the contact angle values obtained by the displacement and conventional methods illustrate the effect of already adsorbed nanoparticles at the oil-rock interface on the contact angles values. The results show that more water-wet substrates are possible to obtain by nanoparticle-fluids. However, a substantial part of wettability alteration by nanoparticles in the water-wet rocks is solely due to the experimental artefacts (trapped nanoparticles in the oil-rock interface) which would not be representative of the real conditions. On the other hand, for oil-wet simple systems, as illustrated in Figure 6b, adding silica nanoparticles into the system by the displacement method had no significant change in contact angle. Whereas, the conventional method of contact angle measurements shows that nanoparticles can alter the wettability of the substrates from oil-wet to intermediatewet condition. Obviously, the changes in the contact angle of the oil-wet conditions is due to experimental artefact, and nanoparticles do not have a significant effect on the wettability of oil-wet rocks.

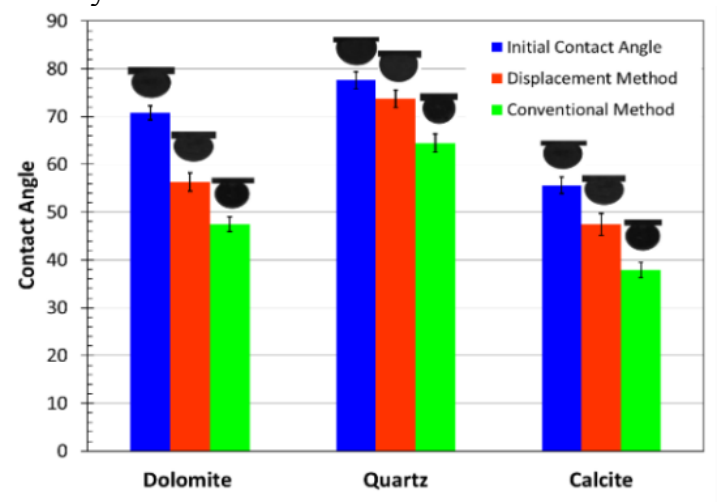

(a) Water-wet condition

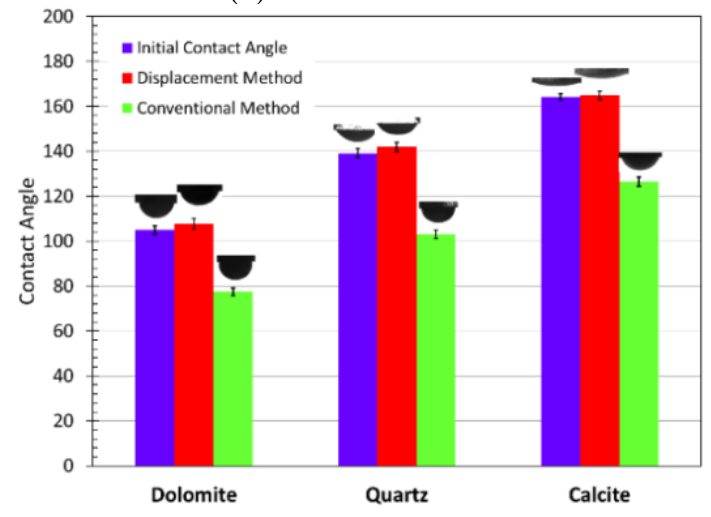

(b) Oil-wet condition

Fig. 6. Wettability alteration due to the presence of silica nanoparticles in simple systems

Similar trends are also observed for complex systems. As shown in Figure 7a, for water-wet conditions, by introducing silica nanoparticles in the system using displacement method, the contact angle value decreases. However, the reduction in contact angle is less than what is obtained using the conventional contact angle method. The contact angle measurements for oil-wet conditions in the complex system again show similar behaviour to the simple system where there is no significant change in contact angle for the oil-wet systems while the contact angles decrease using the conventional method. The difference between conventional and displacement contact angle measurements is summarized in Table 1. 


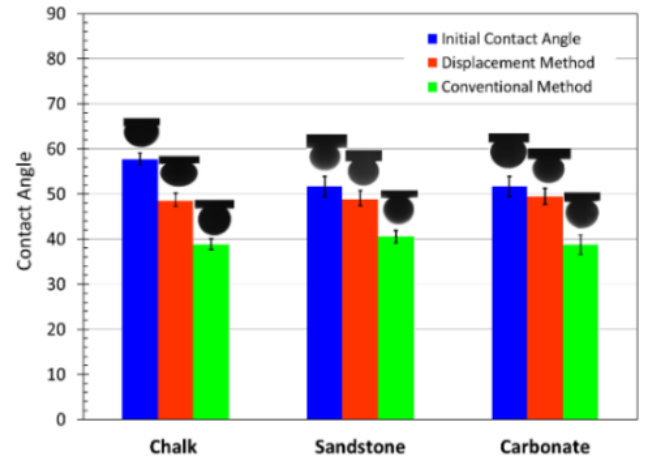

(a) Water-wet condition

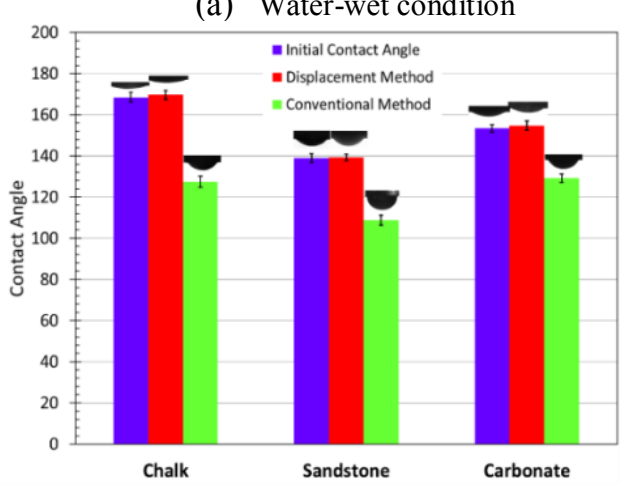

(b) Oil-wet condition

Fig. 7. Wettability alteration due to the presence of silica nanoparticles in complex systems

In water-wet systems, the nanoparticles can interact at the three-phase interface in both the conventional and displacement methods since the surface is water-wet. The exaggerated reduction in contact angle using the conventional method is indicative of the nanoparticles pre-existing at the interface. The marked decrease in contact angles observed for the conventional contact angles measurements on an oil-wet surface indicates that the nanoparticles can interact with the oil covered mineral and rock before an oil droplet is attached. Whereas in the displacement, the nanoparticles have no way of forming a wedge since the oil droplet is in contact with the oil covered mineral or rock.

Based on the disjoining pressure theory for wettability alteration by nanoparticles, for nanoparticles modify the oil-water-rock contact angle, the wedge-film must be formed in the three-phase interface. In the water-wet condition, as shown in Figure 8, there is a wedge-film, therefore, nanoparticles can accumulate in the confinement of oil-water-rock contact region and alter the contact angle by structural disjoining pressure. Furthermore, by introducing the nanoparticles in the system and their adsorption on the oil-water interface, the oil-water interfacial tension and subsequently the capillary pressure decreases. Decreasing the capillary pressure causes the wetting phase (here water) to spread more on the rock surface. Based on the capillary pressure equation $\left(P_{c}=P_{n w}-P_{w}\right)$, the reduction in the capillary pressure means an increase in the pressure of wetting phase, or decrease in the pressure of non-wetting phase, or combination of both. In all cases, the equilibrium between the wetting and non-wetting phases on the rock surface collapses in the direction of excessive pressure from the wetting phase when nanoparticles introduced compared to previously equilibrated condition. Hence, as shown in Figure 8a, the capillary pressure and structural disjoining pressure affect the three-phase contact line in the same direction making the substrate more water-wet. However, under oil-wet conditions, there is no wedgefilm at the three-phase contact line. Hence, the nanoparticles cannot diffuse into the oil-rock interface due to the structural disjoining pressure. On the other hand, as shown in Figure $8 \mathrm{~b}$, the capillary pressure reduction leads to more spreading of the oil phase on the rock surfaces (wetting phase) and consequently slightly shifting the rock surfaces toward oil-wet condition.

Table 1: The difference between conventional and displacement contact angle measurements

\begin{tabular}{|c|c|c|c|c|}
\hline Experiments Type & Rock Type & Oil Type & Initial Contact Angle & $\begin{array}{l}\begin{array}{l}\text { Difference between } \\
\text { measurements methods }\end{array} \\
\end{array}$ \\
\hline \multirow[t]{3}{*}{ Simple water-wet } & Dolomite & Decane & $70.8 \pm 1.5$ & $8.75 \pm 1.9$ \\
\hline & Quartz & Decane & $77.6 \pm 1.8$ & $9.2 \pm 1.9$ \\
\hline & Calcite & Decane & $55.6 \pm 1.7$ & $9.53 \pm 2.3$ \\
\hline \multirow[t]{3}{*}{ Simple oil-wet } & Dolomite & Decane & $104.9 \pm 1.8$ & $30.4 \pm 2.3$ \\
\hline & Quartz & Decane & $139.2 \pm 2.0$ & $39.0 \pm 2.1$ \\
\hline & Calcite & Decane & $164.1 \pm 1.4$ & $38.3 \pm 1.9$ \\
\hline \multirow[t]{3}{*}{ Complex water-wet } & Chalk & Crude oil & $57.8 \pm 1.3$ & $9.6 \pm 1.7$ \\
\hline & Sandstone & Crude oil & $51.7 \pm 2.2$ & $8.2 \pm 2.0$ \\
\hline & Carbonate & Crude oil & $51.7 \pm 2.2$ & $10.6 \pm 1.9$ \\
\hline \multirow[t]{3}{*}{ Complex oil-wet } & Chalk & Crude oil & $168.5 \pm 2.4$ & $42.2 \pm 2.7$ \\
\hline & Sandstone & Crude oil & $138.9 \pm 2.1$ & $30.5 \pm 2.4$ \\
\hline & Carbonate & Crude oil & $153.4 \pm 1.8$ & $25.5 \pm 2.3$ \\
\hline
\end{tabular}

*Average values of three measurements with standard deviations are reported in the table 


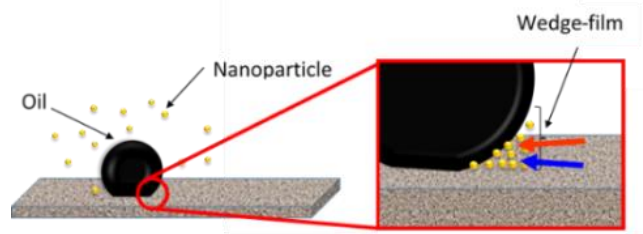

a) Water-wet condition

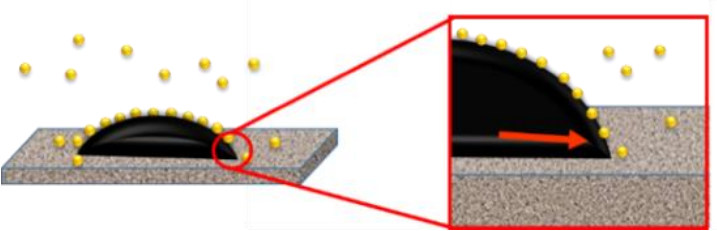

b) Oil-wet condition

Fig. 8. Mechanism of wettability alteration in a) water-wet and b) oil-wet conditions. Red arrow indicates capillary pressure reduction forces and the blue arrow shows disjoining pressure alteration

Effect of the surface and nanoparticles charge: The effect of substrate surface charge and nanoparticles charge on the wettability alteration ability of silica nanoparticles is investigated. To accurately study the mechanism and avoid possible elaborations such as the effect of mineral variety of the rock surface, oil composition, and etc., a simple system of calcite, $\mathrm{NaCl}$ brine, and decane is employed. For $\mathrm{NaCl}$ brine concentrations of $0,1,2$, and $5 \mathrm{wt} \%$, the surface charge of calcite rock, initial contact angle (before introducing nanoparticles), ultimate contact angle (after nanoparticles are introduced with displacement method), and the charge of silica nanoparticles are measured, and the results are illustrated in Figure 9a. By increasing the $\mathrm{NaCl}$ concentration, the surface charge of calcite mineral and silica nanoparticles become less negative. The calcite even switches to a positive absolute charge at $\sim 3 \mathrm{wt} \% \mathrm{NaCl}$. On the other hand, the contact angle measurements reveal that by increasing the salinity, in both cases, with and without nanoparticles, more water-wet substrates can be achieved. However, as shown in Figure 9b, the wettability alteration of calcite substrate due to the presence of nanoparticles decreases with increasing salinity. By increasing the $\mathrm{NaCl}$ concentration, the absolute charge of calcite surface and oil-water interface decreases. Hence, the electrostatic repulsion force between these two surfaces decreases and subsequently the disjoining pressure between the surface and the droplet increases. Furthermore, the excessive salinity compresses the electrical double layer of nanoparticles and consequently reduces the nanoparticlenanoparticle and surface-nanoparticle electrostatic repulsion. Hence, the extra pressure in the wedge-film (structural disjoining pressure) decreases. On the other hand, by increasing salinity and reducing the absolute charge of the nanoparticles, the number of adsorbed nanoparticles at oil-water interface increases. Therefore, the IFT value and capillary pressure decreases. Capillary pressure reduction can lead to more spreading of the wetting phase (in water-wet conditions). However, the impact of structural disjoining pressure outweighs the influence of capillary pressure. Hence, less pronounced wettability alteration is observed by increasing the salinity. The geometries of interface can be different than the simple drop/surface geometries. Hence, further researchers are required to evaluate the capillary pressure/disjoining pressure interactions in 3-D interfaces in pores.
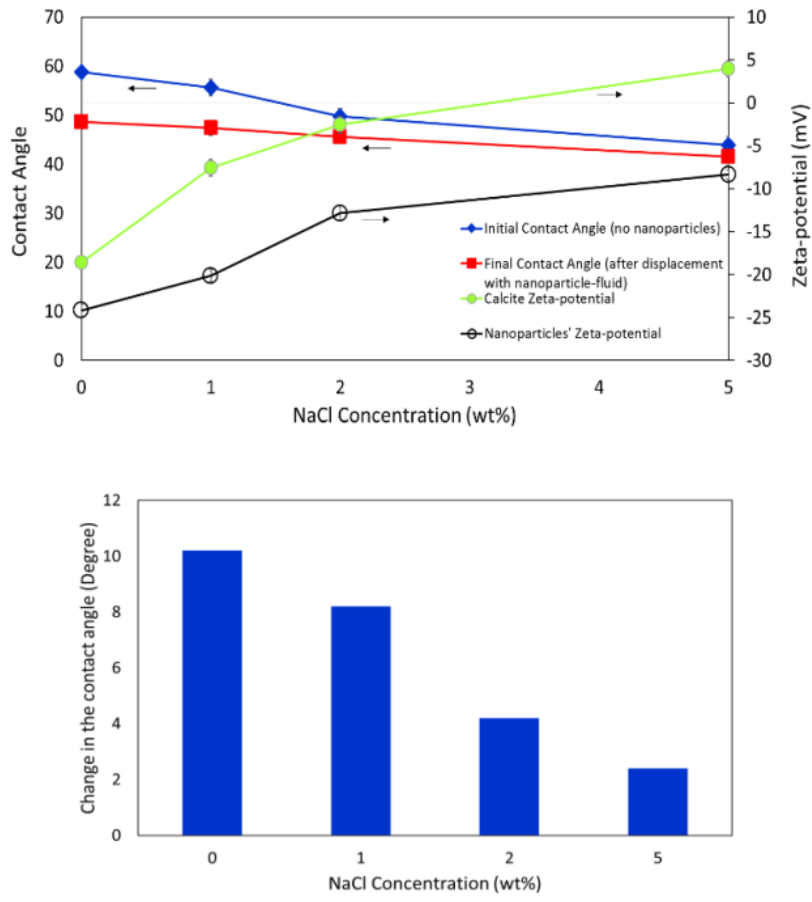

Fig. 9. Top) Effect of the nanoparticles and calcite surface charge on the wettability alteration ability of silica nanoparticles, Bottom) Wettability alteration due to the presence of nanoparticles in various salinities

Migration of nanoparticles through oil phase: The nanoparticles possible migration through the oil phase and its attachment on the rock surface is another uncertainty in the mechanism of wettability alteration due to the presence of nanoparticles. As shown in Figure 10, the primary evaluations with SEM images shows that there is no sign of silica nanoparticles agglomerations on the surface of pure calcite. However, the presence of oil layer on the calcite surface causes the SEM image to not have enough resolution to detect the single nanoparticles (not agglomerations of nanoparticles) on the calcite surface. Hence, more research is required to investigate the diffusion of nanoparticles through oil phase and evaluate the effect of pressure and temperature. 

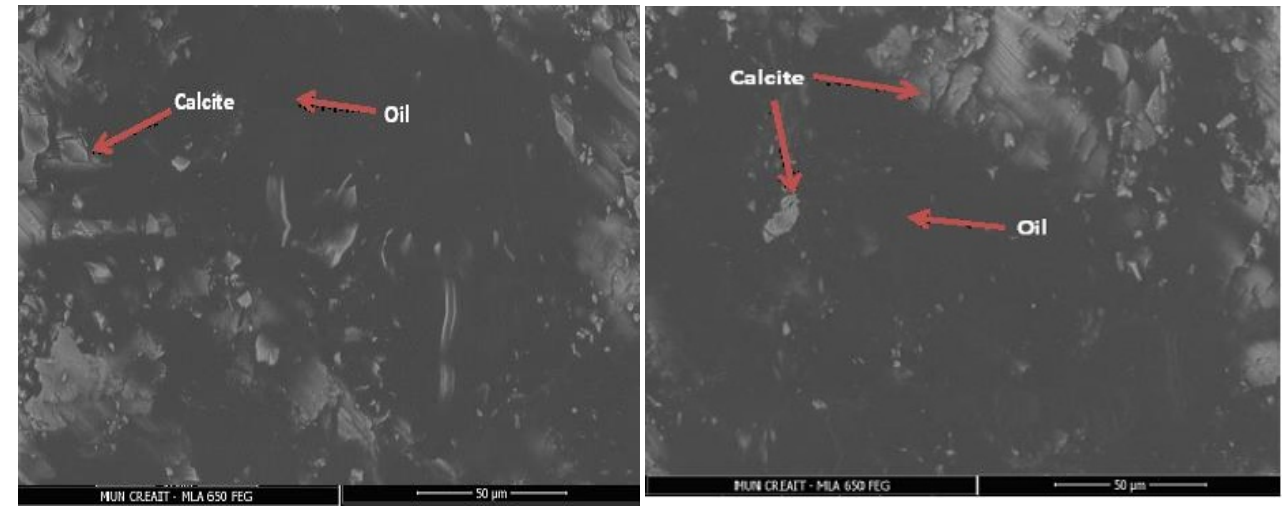

Fig. 10. SEM image of calcite surface before (left) and after (right) contact with nanoparticles

\section{Conclusions}

- A large part of the wettability alteration ability of nanoparticles reported in literature may be attributed to the method of measuring the contact angles where nanoparticles can adsorb on the rock surface before the introduction of the oil droplet. We show that the experimental methodology in how and when the nanoparticles and oil droplet are introduced is important in determining the wettability alteration capability of nanoparticles. If the nanoparticle fluid is in contact with the rock surface before the oil droplet is introduced, the reduction in contact angle may be skewed and overestimated compared to the more realistic situation of the nanoparticle-fluid being introduced into an already established three-phase equilibrium of oil-water-substrate.

- Silica nanoparticles injected in an aqueous based nanoparticle-fluid are shown to reduce the contact angle (make the substrate more waterwet) only when the initial conditions are waterwet. Under oil-wet conditions, there is no significant change in wettability when a nanoparticle-fluid is injected.

- Synergic effect of structural disjoining pressure and capillary pressure reduction is the mechanism of wettability alteration in the waterwet conditions. In the oil-wet conditions, the only possible mechanism is capillary pressure reduction.

- The wettability alteration of calcite substrates in the presence of nanoparticles decreases with increasing salinity due to reduction of nanoparticle-nanoparticle and surfacenanoparticle electrostatic repulsion.

- We did not observe nanoparticles diffusion through an oil film to attach to the rock surface. More investigation is required for conclusive results.

\section{References}

1. Sofla, S.J.D., L.A. James, and Y. Zhang, Insight into the stability of hydrophilic silica nanoparticles in seawater for Enhanced oil recovery implications. Fuel, 2018. 216: p. 559571.

2. Sheng, J., Critical review of low-salinity waterflooding. Journal of Petroleum Science and Engineering, 2014. 120: p. 216-224.

3. Sofla, S.J.D., M. Sharifi, and A.H. Sarapardeh, Toward mechanistic understanding of natural surfactant flooding in enhanced oil recovery processes: the role of salinity, surfactant concentration and rock type. Journal of Molecular Liquids, 2016. 222: p. 632-639.

4. Khezrnejad, A., L. James, and T. Johansen. Water enhancement using nanoparticles in water alternating gas (WAG) micromodel experiments. in SPE Annual Technical Conference and Exhibition. 2014. Society of Petroleum Engineers.

5. Fjelde, I., A.V. Omekeh, and P.E. Haugen. Screening of The Potential for Different Injection Water Compositions To Alter Wettability to More Water-Wet. in SPE Latin America and Caribbean Mature Fields Symposium. 2017. Society of Petroleum Engineers.

6. $\quad$ Rowe, A., et al., Oil detachment from solid surfaces in aqueous surfactant solutions as a function of $\mathrm{pH}$. Industrial \& engineering chemistry research, 2002. 41(7): p. 1787-1795.

7. Kao, R., et al., Mechanisms of oil removal from a solid surface in the presence of anionic micellar solutions. Colloids and Surfaces, 1988. 34(4): p. 389-398.

8. Chengara, A., et al., Spreading of nanofluids driven by the structural disjoining pressure gradient. Journal of colloid and interface science, 2004. 280(1): p. 192-201.

9. Wasan, D.T. and A.D. Nikolov, Spreading of nanofluids on solids. Nature, 2003. 423(6936): p. 156. 
10. Kondiparty, K., et al., Dynamic spreading of nanofluids on solids. Part I: experimental. Langmuir, 2012. 28(41): p. 14618-14623.

11. Miller, C.A. and K.H. Raney, Solubilizationemulsification mechanisms of detergency. Colloids and Surfaces A: Physicochemical and Engineering Aspects, 1993. 74(2-3): p. 169-215.

12. De Gennes, P.-G., Wetting: statics and dynamics. Reviews of modern physics, 1985. 57(3): p. 827.

13. Sefiane, K., J. Skilling, and J. MacGillivray, Contact line motion and dynamic wetting of nanofluid solutions. Advances in colloid and interface science, 2008. 138(2): p. 101-120.

14. Kondiparty, K., et al., Wetting and spreading of nanofluids on solid surfaces driven by the structural disjoining pressure: statics analysis and experiments. Langmuir, 2011. 27(7): p. 3324-3335.

15. Wang, F.-C. and H.-A. Wu, Enhanced oil droplet detachment from solid surfaces in charged nanoparticle suspensions. Soft Matter, 2013. 9(33): p. 7974-7980.

16. Lim, S., et al., The dynamic spreading of nanofluids on solid surfaces-Role of the nanofilm structural disjoining pressure. Journal of colloid and interface science, 2016. 470: p. 22-30.

17. Giraldo, J., et al., Wettability alteration of sandstone cores by alumina-based nanofluids. Energy \& Fuels, 2013. 27(7): p. 3659-3665.

18. Karimi, A., et al., Wettability alteration in carbonates using zirconium oxide nanofluids: EOR implications. Energy \& Fuels, 2012. 26(2): p. 1028-1036.

19. Dai, C., et al., Spontaneous Imbibition Investigation of Self-Dispersing Silica Nanofluids for Enhanced Oil Recovery in LowPermeability Cores. Energy \& Fuels, 2017. 31(3): p. 2663-2668.

20. Ahmadi, M.A. and S.R. Shadizadeh, Induced effect of adding nano silica on adsorption of a natural surfactant onto sandstone rock: experimental and theoretical study. Journal of Petroleum Science and Engineering, 2013. 112: p. 239-247.

21. Joonaki, E. and S. Ghanaatian, The application of nanofluids for enhanced oil recovery: effects on interfacial tension and coreflooding process. Petroleum Science and Technology, 2014. 32(21): p. 2599-2607.

22. Moslan, M.S., et al. Wettability Alteration of Dolomite Rock Using Nanofluids for Enhanced Oil Recovery. in Materials Science Forum. 2016. Trans Tech Publ.

23. Dehghan Monfared, A., et al., Potential Application of Silica Nanoparticles for Wettability Alteration of Oil-Wet Calcite: A Mechanistic Study. Energy \& Fuels, 2016. 30(5): p. 3947-3961.
24. Al-Anssari, S., et al., Wettability alteration of oil-wet carbonate by silica nanofluid. Journal of colloid and interface science, 2016. 461: p. 435 442.

25. Lim, S., et al., Nanofluids alter the surface wettability of solids. Langmuir, 2015. 31(21): p. 5827-5835.

26. Roustaei, A., S. Saffarzadeh, and M. Mohammadi, An evaluation of modified silica nanoparticles' efficiency in enhancing oil recovery of light and intermediate oil reservoirs. Egyptian Journal of Petroleum, 2013. 22(3): p. 427-433.

27. Sripal, E. and L. James, Application of an Optimization Method for the Restoration of Core Samples for SCAL Experiments. Petrophysics, 2018. 59(01): p. 72-81.

28. Saeed Jafari Daghlian Sofla, L.A.J., Yahui Zhang, Understanding the behavior of $\mathrm{H}+$ protected silica nanoparticles at oil-water interface for Enhanced Oil Recovery Implications Journal of Molecular Liquids 2018. 